\title{
Tecnura
}

\section{Los residuos de la construcción y demolición en la ciudad de Cali: un análisis hacia su gestión, manejo y aprovechamiento}

\section{Construction and demolition wastes: analysis of its management and reuse in Cali}

\author{
Rafael Andres Robayo Salazar*, Pedro Enrique Mattey Centeno**, \\ Yimmy Fernando Silva Urrego***, Diana Marcela Burgos Galindo****, Silvio Delvasto Arjona*****
}

Fecha de recepción: 7 de noviembre de 2013

Fecha de aceptación: 5 de diciembre de 2014

Citation / Para citar este artículo: Robayo Salazar, R. A., Mattey Centeno, P. E., Silva Urrego, Y. F., Burgos Galindo, D. M., \& Delvasto Arjona, S. (2015). Los residuos de la construcción y demolición en la ciudad de Cali: un análisis hacia su gestión, manejo y aprovechamiento. Revista Tecnura, 19(44), 157-170 doi:http://dx.doi. org/10.14483/udistrital.jour.tecnura.2015.2.a12

\section{Resumen}

El número de obras de construcción, remodelación y demolición en las ciudades colombianas aumenta de manera constante como consecuencia del crecimiento y desarrollo socioeconómico de la población urbana. Sin embargo, estas prácticas generan grandes volúmenes de residuos que en la actualidad se han convertido en un problema de contaminación ambiental en ciudades como Cali (Colombia). La búsqueda de soluciones integrales que permitan un adecuado manejo, gestión y aprovechamiento de los diferentes materiales que componen los residuos de la construcción y demolición (RC\&D) era impensable hace algunas décadas. No obstante, hoy día es un desafío apremiante para los diferentes sectores de la sociedad que deben encaminar esfuerzos para encontrar soluciones. En este sentido, este artículo de revisión presenta un análisis sobre la situación actual alrededor de este tema en la ciudad mencionada y propone la generación de acciones de acuerdo con las oportunidades y retos necesarios para alcanzar una gestión total de los RC\&D. El análisis de esta problemática podría ayudar a orientar futuras investigaciones sobre el tema, además de promover su prioridad en la agenda pública, generando así las condiciones necesarias para que la actividad del reciclaje de los RC\&D se convierta en una posibilidad viable en las ciudades colombianas a partir de la experiencia obtenida.

Palabras clave: aprovechamiento, gestión de los RC\&D, materiales de construcción, residuos de la construcción y demolición (RC\&D).

\section{Abstract}

The amount of construction, remodeling and demolition activities in the Colombian cities increases constantly due to growth and economic development of

* Ingeniero de Materiales, estudiante de doctorado en Ingeniería con énfasis en Ingeniería de los Materiales. Universidad del Valle, Cali, Colombia.Contacto: rafael.robayo@correounivalle.edu.co

** Ingeniero Metalúrgico, magíster en Ingeniería de los Materiales, estudiante de doctorado en Ingeniería con énfasis en Ingeniería de los Materiales. Universidad del Valle, Cali, Colombia. Contacto: pedromattey@gmail.com

*** Ingeniero de Materiales, estudiante de maestría en Ingeniería de los Materiales. Universidad del Valle, Cali, Colombia. Contacto: yimmy. silva@correounivalle.edu.co

**** Ingeniero de Materiales, estudiante de doctorado en Ingeniería con énfasis en Ingeniería de los Materiales. Universidad del Valle, Cali, Colombia. Contacto: diana.burgos@correounivalle.edu.co

***** Ingeniero químico, magíster en Ingeniería Civil y Gestión de Materiales, doctor en Ingeniería de Nuevos Materiales y sus tecnologías de fabricación, profesor titular de la Universidad del Valle. Cali, Colombia. Contacto: silvio.delvasto@correounivalle.edu.co 
the urban population. However, these practices create big quantities of debris that have currently become an environmental problem in cities such as Cali (Colombia). The search for integral solutions that allow for an adequate management and reuse of the different materials that form construction and demolition debris was unthinkable some decades ago. Nonetheless, nowadays it is an urgent challenge for different sectors in the society that must join efforts to find solutions. Regarding this, the present article shows an analysis about the currently situation of the construction debris management in the aforementioned city and proposes different actions according to the necessary opportunities and challenges to achieve an integral management and reuse of these materials. The analysis of this topic could be a guide for future research, as well as promote its priority in the public agenda, creating the necessary conditions for the recycling process of the construction and demolition wastes to become a viable possibility in the Colombian cities through the obtained experience. Keywords: construction materials, construction waste, demolition waste, management and recycling.

\section{INTRODUCCIÓN}

Los residuos de la construcción y demolición (RC\&D) o escombros como son llamados en algunos países latinoamericanos, son en general materiales de desecho generados durante la ejecución de un proyecto de obra civil; incluyendo estructuras y materiales rechazados, materiales que han sido descartados, materiales que han sido utilizados y se han deteriorado, e incluso aquellos derivados de actividades de excavación y limpieza del lugar al finalizar la obra, entre otros (Torgal \& Jalali, 2011) (Oikonomou, 2005) (Poon \& Chan, 2007) (Lin et al., 2004) (Limbachiya et al., 2004) (Adam \& Kurama, 2013).

Actualmente en la ciudad de Santiago de Cali se producen cerca de 2480 metros cúbicos de estos residuos por día, cifra que en un año logra alcanzar un millón de metros cúbicos; esto de acuerdo con el número de habitantes Ilega a ser 0,5 metros cúbicos de RC\&D por persona, índice que resulta alarmante y sitúa a la mencionada ciudad en el segundo puesto de las capitales colombianas con mayor generación de escombros, superada por la capital del país, Bogotá, que con un promedio de 12 millones de toneladas de RC\&D al año ocupa el primer lugar a nivel nacional (Castaño et al., 2013). Por esta razón la municipalidad en los últimos años ha optado por buscar soluciones integrales que permitan mitigar la problemática social y ambiental derivada de la alta generación de RC\&D originada por el desarrollo urbanístico de los últimos años (DAGMA, 2011). Todas estas alternativas orientadas a lograr una adecuada gestión y manejo de estos residuos que lleve al aprovechamiento sostenible de gran parte del volumen que actualmente se genera en la ciudad.

Este artículo muestra la situación actual de la ciudad de Santiago de Cali en torno a los RC\&D y analiza las deficiencias que presentan los procesos actuales de manejo y gestión de estos residuos. Con base en lo anterior, este articulo propone acciones que lleven a poner en marcha un proceso estandarizado que permita el reciclaje de los RC\&D, avalado por normas técnicas y una legislación que asegure su sostenibilidad.

\section{ANTECEDENTES}

La construcción es una de las actividades industriales con mayor demanda en Colombia, que se consolida por su gran dinamismo como uno de los sectores más importantes y de mayor incidencia en el desarrollo económico del país. Sin embargo, una de las consecuencias que se derivan de esta actividad es la generación de volúmenes considerables de RC\&D. En la actualidad, Colombia cuenta con desarrollo legislativo enmarcado en la "Política Nacional para la Gestión de Residuos Sólidos" que establecen los parámetros mínimos que 
se deben llevar a cabo para un adecuado manejo y gestión de los residuos sólidos y RC\&D en el territorio nacional; como es el caso de la Resolución 541 de 1994 y la Ley 1259 de 2008 (Congreso de la República, 2008). Sin embargo, muchas de estas leyes han sido formuladas aprovechando la legislación de otras naciones más desarrolladas y su aplicación en el territorio nacional ha resultado infructuosa en razón de las diferencias existentes, como, por ejemplo, la cultura ambiental de sus habitantes (Hincapíe \& Aguja, 2003).

Adicionalmente cada municipio ha desarrollado su propio marco legal para la gestión y en algunos casos el aprovechamiento de los RC\&D. En Bogotá, por ejemplo, la implementación de la Resolución No. 2397 de 2011 de la Secretaría Distrital de Ambiente permite la regulación técnica del tratamiento y/o aprovechamiento de los escombros en el distrito capital, y establece que a partir del año 2012 las entidades que desarrollen obras de infraestructura en la ciudad deberán incluir la utilización de materiales y elementos elaborados a partir del reciclaje de RC\&D en un porcentaje no inferior a $10 \%$ del total de metros cuadrados construidos para las entidades públicas y $5 \%$ para las entidades privadas. Además se establece que cada año dicho porcentaje aumentará en $5 \%$ hasta alcanzar un mínimo de 25\% (Alcaldía Mayor de Bogotá, 2011).

En Medellín, la aprobación del "Plan de Gestión Integral de Residuos Sólidos" (PGIRS, Acuerdo 062 de 2009) estableció una política pública para la gestión de los RC\&D generados en la capital de Antioquia; sin embargo, un proyecto de reglamentación de este Acuerdo Municipal tiene como objetivo el incluir un sistema de aprovechamiento similar al propuesto en Bogotá mediante la Resolución No. 2397 de 2011, para el cual se propone el uso en obras civiles de un porcentaje no inferior a $5 \%$ en elementos no estructurales elaborados con materiales obtenidos a partir de RC\&D, además del aumento anual de $2 \%$ hasta llegar a un máximo de 15\% (Consejo de Medellín, 2009) (Alcaldía Mayor de Bogotá, 2012) (Alcaldía
Mayor de Bogotá, 2011) (Alcaldía de Santiago de Cali, 2012).

Por otro lado, en el caso de estudio el Decreto Municipal No. 0291 de 2005 regula la gestión integral de los RC\&D y contiene las herramientas necesarias para que la Municipalidad adelante un buen manejo y regulación de estos residuos, incluyendo su generación, recolección, transporte, transferencia, aprovechamiento y disposición final. Así mismo establece, según el Artículo 59 del mencionado decreto, la creación del Comité Municipal de Escombros, como un espacio interinstitucional e intersectorial articulador de las acciones que desarrollen en el Municipio en esta temática (Alcaldía de Santiago de Cali, 2005) (DAGMA, 2012). En la figura 1 se presentan los antecedentes del marco jurídico del manejo de los RC\&D en la ciudad de Cali; se puede observar que la mayoría de disposiciones tienen como finalidad la regulación del manejo de los RC\&D desde su generación hasta su disposición final (Congreso de Colombia, 2000) (Alcaldía Mayor de Bogotá, 2002) (Congreso de Colombia, 2002) (Alcaldía de Santiago de Cali, 2004) (Alcaldía de Santiago de Cali, 2009).

Además de la legislación propia del país y el Municipio creada para la gestión de los RC\&D, diferentes sectores de la sociedad, incluyendo la academia, han desarrollado numerosas propuestas tecnológicas de aprovechamiento que aprueban la reutilización sostenible de los RC\&D en nuevos materiales de construcción con excelentes resultados. No obstante, la mayoría de estos esfuerzos resultan infructuosos y no trascienden debido principalmente a que en Colombia no existen normas técnicas basadas en estudios científicos que especifiquen, regulen y avalen la reutilización de los RC\&D en diferentes sectores de la industria de la construcción, lo cual fue verificado por los autores en diversas fuentes de información. Y que a su vez brinden la suficiente seguridad al consumidor, que a partir de estos materiales se puedan desarrollar productos de excelente calidad y durables a través del tiempo. 
Decreto 2811 de 1974: Código de los Recursos Nacionales. Art. 37: Responsabilidad de los municipios en la gestión de sus residuos.

Ley 99 de 1993: Creación del SINA. Art. 66: Responsabilidades de las autoridades municipales en el control y seguimiento de los residuos.

Resolución 541 de 1994: Cargue, Descargue, Transporte, Almacenamiento y disposición Final de Escombros. Art. 3: Requerimientos para la gestión integral y disposición final de escombros.

Ley 142 de 1994 - Decreto 605 de 1996: Presentación del Servicio Público y Aseo. Art. 104: Restricciones de los generadores. Art. 113: Competencias de las autoridades de policía en la aplicación de sanciones y correctivos.

Ley 632 de 2000: Contribución de Solidaridad en la Autogeneración. Art. 9: Esquemas de prestación del servicio público domiciliario y aseo.

Decreto 1713 de 2002: Gestión Integral de los Residuos Sólidos. Art. 44: Responsabilidades de los generadores de escombros.

Ley 769 de 2002: Código Nacional de Tránsito. Art. 102: Responsabilidad de las autoridades de tránsito en la vigilancia, control y sanciones.

Resolución 1042 de 2003: Metodología para la Elaboración de los Planes de Gestión Integral de Residuos Sólidos.

Decreto 0475 de 2004: Adopción del Plan de Gestión Integral de Residuos Sólidos de Cali (PGIRS) Programa para la gestión integral de escombros de Santiago de Cali.

Decreto No. 0291 de 2005: Por medio del cual se regula la gestión integral de escombros en el municipio de Santiago de Cali. Artículo 16. Requerimiento para los Vehículos que Transportan de Escombros. Artículo 18: Requerimientos para la gestión y Transporte de Escombros. Artículo 19: Restricción para el transporte de escombros en vías y horarios. Artículo 28: Estaciones de Transferencia y Centros de Acopio. Artículo 59: Creación del Comité Municipal de Escombros.

Decreto No. 0059 de 2009: por medio del cual se adoptan los manuales para la implementación de programas de gestión integral de residuos sólidos.

Ley 1259 de 2008 y Acuerdo 0327 de 2012: Comparendo Ambiental; Instrumento de control y sanción para los infractores de las normas de aseo, limpieza y recolección de escombros.

Figura 1. Antecedentes del marco normativo para el manejo de RC\&D en la ciudad de Cali.

Fuente: elaboración propia.

\section{DIAGNÓSTICO DE LA PROBLEMÁTICA ACTUAL DE LOS RC\&D EN COLOMBIA}

Actualmente en Colombia la gestión de los RC\&D tiene como desenlace el vertimiento de estos residuos en escombreras, manejo que en contadas ocasiones es controlado; sin embargo, la disposición de estos residuos en sitios no autorizados es hoy día una práctica muy frecuente en la mayoría de las ciudades colombianas, siendo hasta ahora la opción más sencilla de desechar estos residuos; no obstante, esta resulta ser una opción cíclica, económicamente inviable, no definitiva y con mayor impacto ambiental, pues el inminente agotamiento de los vertederos hace necesaria la búsqueda constante de nuevos lugares para la 
disposición de estos residuos. Unido a esto, la escasez de cualquier alternativa de aprovechamiento que esté avalada técnicamente hace que el manejo de los RC\&D resulte totalmente ineficiente, ya que en la actualidad estos no representan valor productivo alguno para la sociedad y se convierten en una problemática ambiental y social por su difícil manejo (Serrano \& Ferreira, 2009).

Uno de los ejemplos que mejor ilustran esta problemática es el de la ciudad de Bogotá, en donde según la Unidad Administrativa Especial de Servicios Públicos (UAESP) se producen cerca de 12 millones de toneladas de RC\&D al año, de las cuales solo 333.000 toneladas tienen un manejo adecuado por parte de la UAESP, el resto presentan un manejo que es considerado ilegal, al ser vertidas de manera no controlada en alrededor de 94 sitios no autorizados, causando problemas sanitarios y ambientales a la ciudad; de ahí que la generación e implementación de leyes dirigidas a la regulación de los RC\&D, como la Resolución No. 2397 de 2011, sea una prioridad en la gestión pública de la ciudad (El Tiempo, 2013) (Castaño et al., 2013) (UAESP, 2012) (Alcaldía Mayor de Bogotá, 2012) (El Tiempo, 2012).

Por otra parte, en Medellín los desechos resultantes de las actividades de construcción alcanzan una cifra cercana a 2.400 metros cúbicos; por esta razón la administración municipal, por medio de las Empresas Varias, ha implementado acciones como la recolección domiciliaria de los RC\&D para promover el manejo adecuado de estos residuos. Además, continúa en la búsqueda de nuevos centros de acopio temporal para los mismos, así como la intención de poner en marcha todo un programa de aprovechamiento de los $R C \& D$ recolectados de manera formal en la ciudad con base en el PGIRS aprobado mediante el Acuerdo Municipal del Concejo de Medellín No. 062 de 2009 (Bedoya \& Gonzales, 2003) (Hincapíe \& Aguja, 2003).

En general, la problemática de los RC\&D en las diferentes ciudades colombianas es muy similar; sin embargo, este comportamiento está en función del número de sus habitantes, siendo más crítico en ciudades que superan 2 millones de habitantes, como Bogotá, Medellín y Cali. No obstante, en ciudades más pequeñas esta problemática es cada vez más notoria debido al gran dinamismo que presenta el sector de la construcción. Tal es el caso de Cartagena, donde en los últimos años la actividad de la construcción ha aumentado considerablemente y se están generando anualmente alrededor de 190.000 metros cúbicos de RC\&D, cuya disposición se hace en sitios que en su mayoría no son autorizados; de acuerdo con esta situación, el municipio requiere actualmente una escombrera con una capacidad de 2.900.000 metros cúbicos para disponer lo generado hasta el año 2018. Un caso similar al de Cartagena se está presentando en Ibagué, en donde se producen anualmente alrededor de 500.000 metros cúbicos de RC\&D que generan problemas a nivel ambiental y social en diferentes sectores de la capital tolimense, razón por la cual la alcaldía de este municipio adelanta planes que ayuden a mitigar este problema y para ello aprobó la Resolución 2475 de 2010, que regula y controla la disposición final de estos residuos y sanciona a quienes incumplan su contenido (Eijaiek et al., 2011).

\section{RC\&D EN LA CIUDAD DE CALI}

\section{Generación de RC\&D en la ciudad de Cali}

Como se mencionó antes, la capital vallecaucana genera actualmente un promedio de 2480 metros cúbicos diarios de RC\&D. De este volumen, cerca de 76,6\% (1900 metros cúbicos) es aportado por las constructoras y obras públicas en lo que se denomina "la generación formal" y 23,4\% (580 metros cúbicos) restante son aportados por construcciones y remodelaciones particulares, y el Ilamado "sector informal" (Sánchez et al., 2013). Los mayores productores de RC\&D son las constructoras y las obras públicas; por esta razón, el desarrollo de proyectos urbanísticos como las 21 mega-obras y la construcción del Masivo Integrado de Occidente (MIO) han provocado un incremento significativo del volumen de RC\&D generados anualmente en la ciudad de Cali desde 
el año 2009 (figura 2), cuando se encontraban en ejecución las etapas finales de dichos proyectos. A partir de 2011 hasta la actualidad, la generación de RC\&D se ha mantenido constante; sin embargo, la proyección puede variar para los próximos años, ya que el municipio tiene planeada la ejecución de una segunda fase de las mega-obras, con lo cual se espera que la cifra de generación de RC\&D se vuelva a incrementar (Alcaldía de Santiago de Cali, 2012) (El Tiempo, 2012).

\section{Gestión y manejo de los RC\&D en la ciudad de Cali}

Actualmente el manejo de los RC\&D en la ciudad de Cali se divide en dos sectores; formal (constructoras) e informal (particulares); en este sentido existen tres alternativas para transportar los RC\&D desde su lugar de origen hasta el sitio de disposición final o estación de transferencia, entre los cuales se encuentran: los volqueteros, los carretilleros y las empresas públicas de aseo (EMAS, Promoambiental, Ciudad Limpia, etc.) (El Tiempo, 2013) (Alcaldía de Santiago de Cali, 2005).
Se estima que dentro del sector informal cerca de $40 \%$ utiliza los operadores de aseo como el medio de transporte para sus residuos, esta práctica es quizás la que presenta mayor beneficio para el manejo de los RC\&D en la ciudad debido a que estas empresas se rigen por las leyes establecidas por el municipio y disponen directamente estos residuos en las estaciones de transferencia de manera semi-controlada. No obstante, $60 \%$ restante hace uso de los carretilleros pagando un valor que oscila entre $\$ 15.000$ y $\$ 25.000$ para deshacerse de sus RC\&D sin importar el destino, ya que al no representar estos materiales un beneficio económico para estos transportadores y en algunos casos las grandes distancias hacia las estaciones de transferencia, sumado a la escasez de cultura ambiental, justifica para estas personas el vertimiento indiscriminado de los RC\&D en lugares no autorizados, tal como se puede observar en la figura 3. Por esta razón es fundamental incluir a los carretilleros en el proceso de reciclaje para motivar en ellos el adecuado manejo de los escombros de la construcción, y de este modo los desechos provenientes del sector informal también puedan ser aprovechados

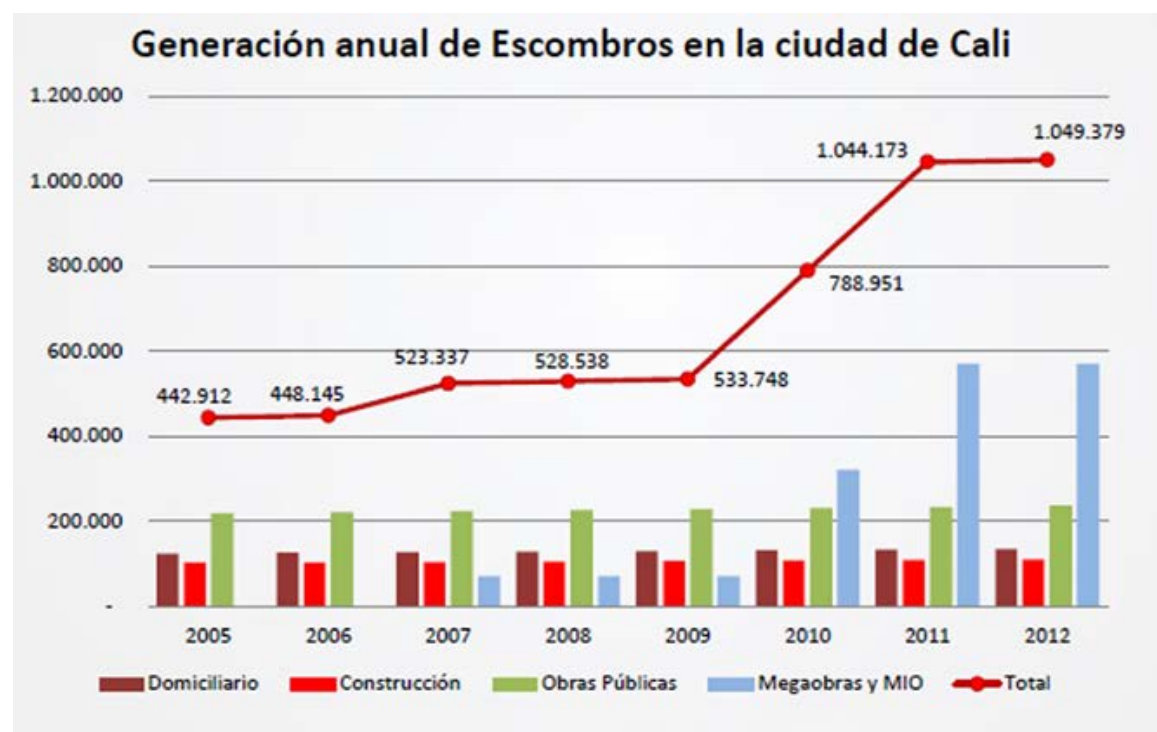

Figura 2. Generación anual (en toneladas) de RC\&D en Santiago de Cali en los últimos años.

Fuente: (DAGMA, 2012). 
dentro de un proceso de reutilización, contribuyendo de manera activa a que este tipo de residuos dejen de ser considerados una problemática ambiental para convertirse en una oportunidad laboral para la sociedad (Sánchez et al., 2013).
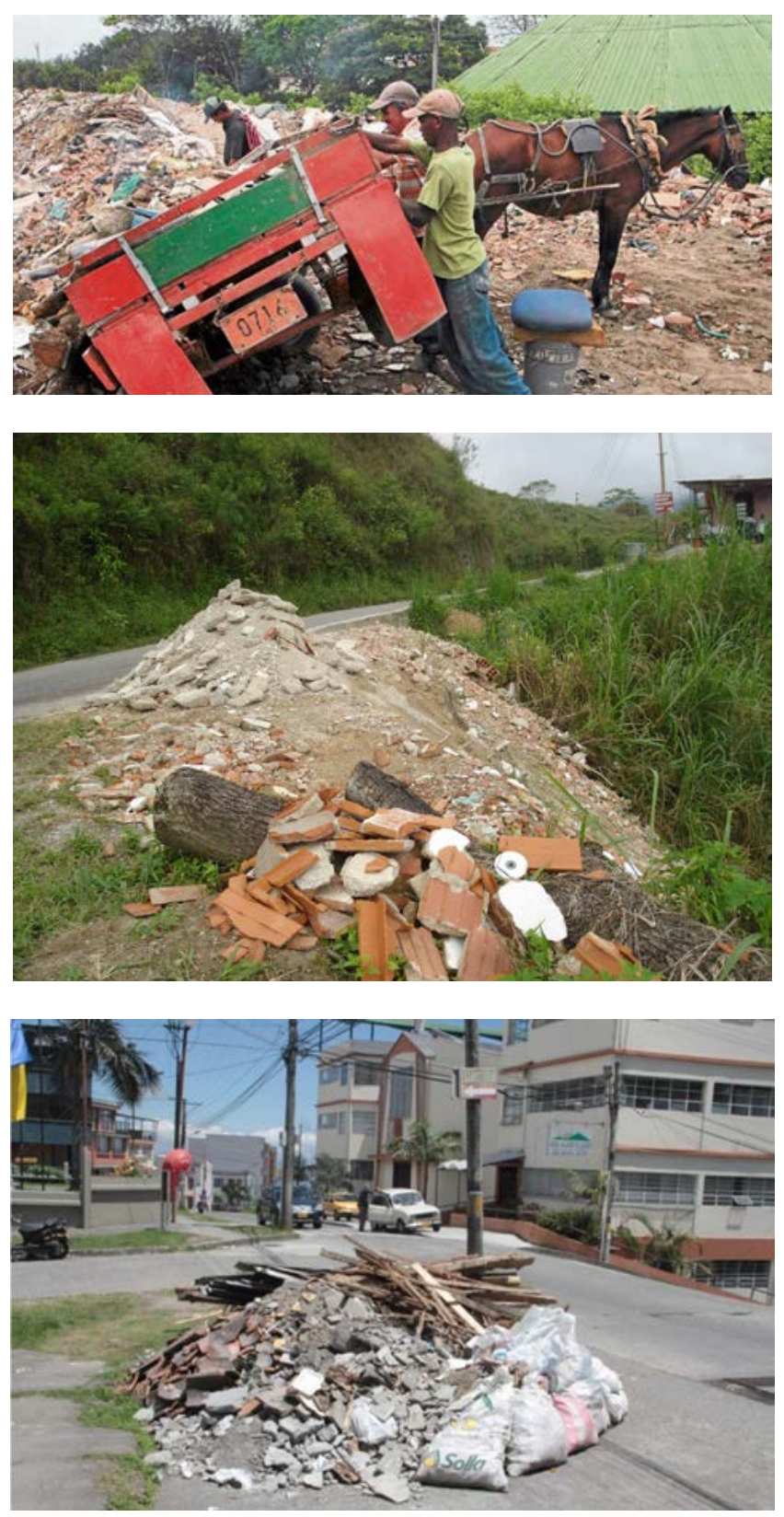

Figura 3. Vertimiento ilegal de RC\&D en Santiago de Cali.

Fuente: (Diario ADN, 2013).
Algunas constructoras (sector formal) prefieren contratar los volqueteros para transportar sus residuos, pero estos al no estar vinculados legalmente a una empresa en ocasiones los disponen de manera inadecuada, razón por la cual en el año 2013 el DAGMA adelantó un proceso sancionatorio a cerca de 42 constructoras en la ciudad (Sánchez et al., 2013) (DAGMA, 2013). Por otra parte, las demás constructoras contratan a una empresa privada transportadora o aprovechadora de escombros, que en el caso de la ciudad de Cali es PROGEA DEL VALLE, quienes separan y disponen los residuos de manera controlada en áreas en donde antiguamente se explotaba material arcilloso en el municipio de Candelaria; no obstante, esta práctica le genera al municipio un costo adicional al ya definido para el manejo y gestión de los mismos.

En general, se calcula que a la ciudad le cuesta el manejo de 1 metro cúbico de RC\&D aproximadamente $\$ 20.577$ pesos, en los cuales se incluyen los costos de transporte, cargue y descargue y disposición final. Sin embargo, a este valor se deben sumar otros que son difíciles de cuantificar pero que no dejarán de ser unos gastos para el municipio, como son los costos asociados a la limpieza periódica de las estaciones de transferencia, los costos de los operadores y su control logístico, y el costo del impacto ambiental que le genera el inadecuado manejo y gestión de los RC\&D. Lo expuesto justifica el reciclaje de estos materiales como una oportunidad para la ciudad y una alternativa económicamente viable con respecto a la cuantificación de todos los gastos que representa la gestión que se realiza actualmente alrededor de los escombros (DAGMA, 2011).

Otra de las causas del inadecuado manejo de los RC\&D que se analiza a diario en la ciudad es que el municipio no cuenta con un sitio de disposición final para estos residuos, esto sumado a que muchos de los carretilleros y empresas llevan los desechos de manera ilegal a sitios no autorizados, como parques, zonas verdes y vías, hace que la generación de focos de contaminación alrededor de la ciudad traiga como consecuencia problemas de 
salubridad que afectan de manera directa a la población en general (DAGMA, 2014) (El País, 2008) (Diario El Pueblo, 2013). El DAGMA ha reportado que en la ciudad hay aproximadamente 109 botaderos de basura crónicos; entre ellos, 55 son sitios en donde se arrojan RC\&D, de los cuales 36 siguen activos y 19 han sido intervenidos parcialmente por las autoridades de aseo, salud pública y medio ambiente de la ciudad, tal como se puede observar en la figura 4 (Sánchez et al., 2013) (Alcaldía de Santiago de Cali, 2012).
A pesar de todos los esfuerzos, el manejo que se da hoy a los RC\&D en la ciudad de Cali continúa siendo inapropiado, por lo que estos terminan siendo tratados como un desecho y contaminados desde la fuente con otros materiales, e incluso con materia orgánica (figura 5). Esta mala práctica hace imposible llevar a cabo la labor de reciclaje y aprovechamiento de los RC\&D (actividades que en la ciudad son poco habituales) (Yaprak et al., 2011). Por esta razón, antes de pensar en el aprovechamiento de estos materiales es

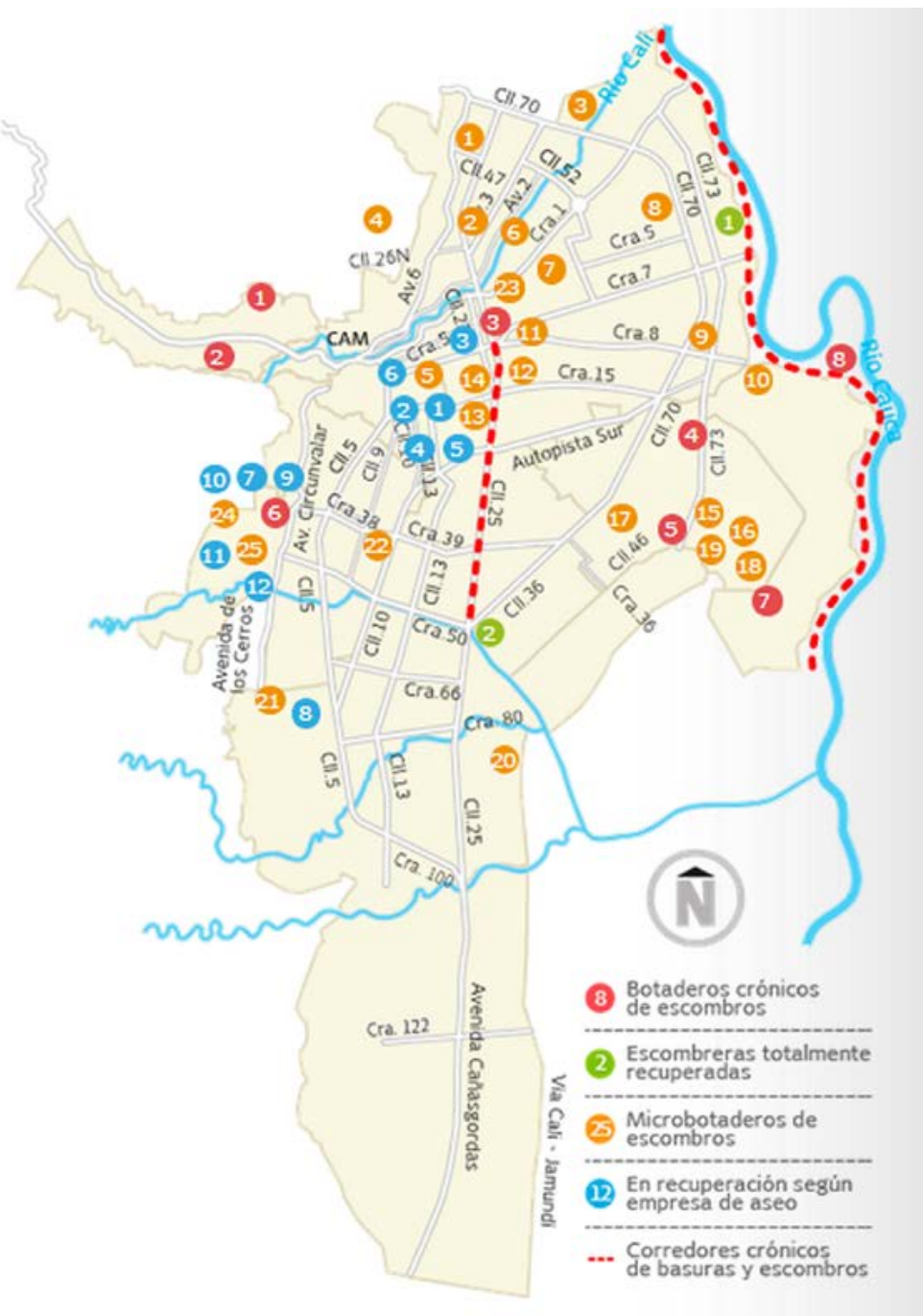

Figura 4. Distribución de los sitios de disposición de los RC\&D en Santiago de Cali.

Fuente: (Sánchez et al., 2013). 
indispensable que el plan de gestión y manejo de los RC\&D de la ciudad se cumpla a cabalidad desde la fuente que los origina hasta el lugar de disposición ya sea final o de transferencia. Esto con el fin de que estas se conviertan en un centro de acopio en donde se realicen labores de selección y separación de los materiales aprovechables, para posteriormente distribuirlos a los diferentes procesos de reutilización correspondiente a cada tipo de residuo.
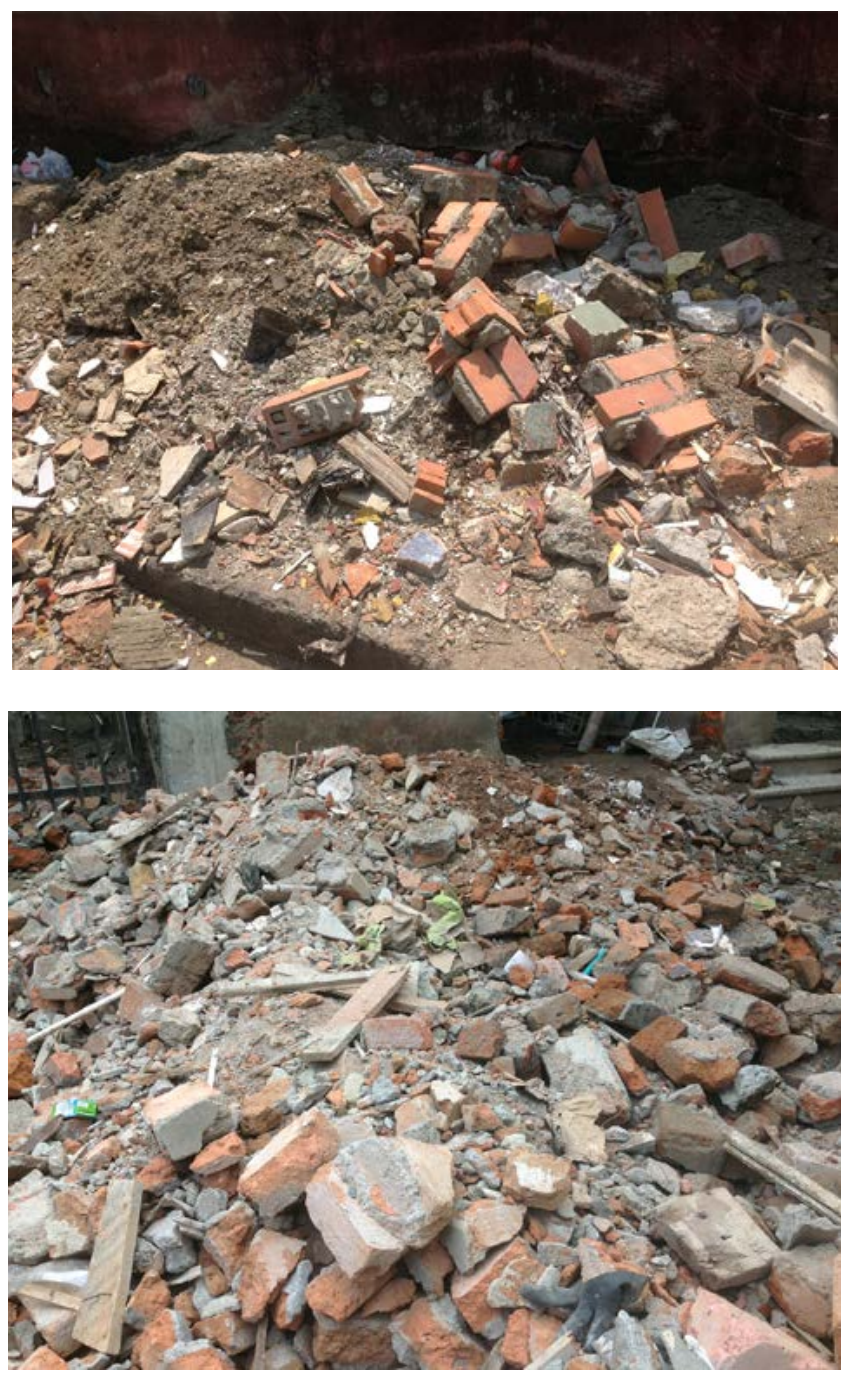

Figura 5. Vertimiento no autorizado de RC\&D contaminados en Santiago de Cali.

Fuente: Elaboración propia.

\section{Barreras para el buen manejo y reciclaje de los escombros en Santiago de Cali}

Se han identificado algunas barreras o dificultades para la implementación de los procesos de reciclaje de los RC\&D y para el cumplimiento de la legislación actual, entre las cuales se encuentran: la falta de cultura ambiental y el comportamiento poco cívico en la población de Santiago de Cali, los bajos incentivos financieros para las empresas que efectúan buenas prácticas para el manejo de sus escombros, la escasa prioridad ambiental en la agenda pública, un mercado competitivo, la falta de conocimiento sobre el reciclaje de estos materiales, y la escasa articulación en la cadena del reciclaje de los carretilleros, volqueteros y operadores encargados de la recolección de los RC\&D (Rakshvir \& Barai, 2006) (Sriravindrarajah et al., 2012).

Sin embargo, la barrera que quizá tiene mayor influencia y hace infructuosos los procesos de reciclaje de RC\&D en Santiago de Cali y en la mayoría de ciudades colombianas es la inexistencia de normas técnicas que hayan sido avaladas por estudios e investigaciones científicas y que permitan el uso de materiales obtenidos a partir de escombros en obras de construcción civil y, sirvan a su vez como medio para brindar seguridad al consumidor de la calidad del producto obtenido. La falta de estas herramientas hace necesario el desarrollo de estudios y acciones que se enfoquen en el reciclaje de este tipo de materiales, para así establecer todo el soporte técnico y científico que permita generar normas técnicas y las medidas legislativas necesarias para incentivar y avalar la reutilización, reciclaje y aprovechamiento de los RC\&D en la ciudad.

\section{BENEFICIOS DE GESTIONAR Y RECICLAR LOS RC\&D}

Los beneficios al manejar adecuadamente y reutilizar los RC\&D son diversos; por ejemplo, desde el punto de vista ambiental se generaría el mayor impacto, pues se reducirían los focos de contaminación alrededor de toda la ciudad y los problemas 
de salubridad relacionados con esto (aire, suelo y agua contaminada) (Aldana \& Serpell, 2012) (Janssen, 2005) (Thomas et al., 2013). En cuanto el aspecto económico se podría lograr una reducción considerable en los costos de manejo y disposición final por parte de las autoridades municipales encargadas, además de una reducción en los gastos asociados a la limpieza por el vertimiento inapropiado de estos residuos alrededor de toda la ciudad (Valdéz \& Rapimán, 2007) (Muller, 2005) (Salem et al., 2003). Los beneficios sociales incluyen la disminución de botaderos de escombros no autorizados, reduciendo los riesgos ambientales y evitando la desvalorización de los predios cercanos a estos lugares. Por otra parte, al clasificar los RC\&D podrían ser transformados en agregados reciclados de concreto (ARC) tanto gruesos como finos para la producción de mezclas de concreto y mortero, bases y sub-bases granulares y elementos prefabricados (Malasev et al., 2012) (Deshpande et al., 2011) (Xiao et al., 2012) (Shima et al., 2005) (Vázquez, 2005) (Lederle \& Hiller, 2013). Se generaría así otro beneficio en cadena desde el punto de vista ambiental porque se lograría reducir la demanda y extracción de materias primas naturales como la grava y la arena para el uso en el sector de la construcción (Xiao et al., 2012) (Seddik et al., 2012) (Yang et al., 2008).

\section{CONCLUSIONES}

Este artículo ha puesto de manifiesto las fuentes y causas de la generación de los RC\&D en la ciudad de Cali, así como los intentos y estrategias que se han generado al nivel nacional y local para su reducción y control; sin embargo, se hace notoria la falta de integración de los municipios en el campo de la gestión de los RC\&D al nivel nacional. En este sentido, la articulación de los hallazgos individuales obtenidos en los diferentes procesos que se llevan a cabo se hace indispensable, de tal forma que al unificarlos se promueva la generación de proyectos con el apoyo de la academia que apunten a la generación de herramientas prácticas que solucionen paulatinamente los problemas de gestión, manejo y aprovechamiento de los RC\&D en los diferentes municipios de Colombia.

Es necesario el desarrollo y la integración de múltiples estudios e investigaciones sobre el aprovechamiento de los diferentes materiales que componen los RC\&D con el fin de adquirir todo el soporte técnico y científico necesario para la formulación de toda una legislación y normatividad que avalen el uso de los materiales obtenidos a partir del procesamiento de los RC\&D en las ciudades colombianas. En primera instancia, estas normas deberán establecer los procedimientos a seguir para el procesamiento adecuado de los RC\&D, así como las especificaciones que deberán cumplir, y los procesos y las proporciones óptimas de incorporación de los materiales obtenidos para poder ser utilizados en el sector de la construcción.

La generación de normas que avalen el uso de los RC\&D debe ir acompañada con la formulación de medidas legislativas que promuevan su utilización e incorporación en obras de infraestructura; estas deberían incentivar o favorecer a aquellas entidades que las cumplan y sancionar a las que no las acaten. De esta forma se estará no solo promoviendo la reutilización y el reciclaje de estos materiales sino también una conciencia ambiental, dirigida al adecuado manejo de los RC\&D, con el fin de no contaminarlos en la fuente para su posterior aprovechamiento.

Al darle un correcto aprovechamiento a los RC\&D se les asignaría directamente un valor agregado, por lo que aquellas personas que trabajan alrededor de estos materiales serían favorecidas al lograr ser articulados en toda la cadena de reciclaje. Se obtendría así una cultura de recolección, manejo y vertimiento adecuado de los escombros, lo que permitiría que los $R C \& D$ representen un ingreso económico adicional al ya obtenido por transportarlo hacia los sitios autorizados. De esta manera se disminuirían los focos de contaminación generados por el sector informal que en este caso es el más complicado de controlar, sin llegar a poner en riesgo el derecho al trabajo de estas 
personas. Además, teniendo en cuenta que en todo proceso de selección y separación de materiales la mano de obra se hace indispensable, y considerando que estas prácticas deben empezar por su fuente, esta se convierte en una alternativa adecuada para que la parte informal se integre a la cadena del reciclaje de estos residuos.

Es evidente la necesidad actual que tiene la ciudad de Cali de contar con una escombrera; sin embargo, también es fundamental contar con pequeñas estaciones de transferencia en algunos sectores de la ciudad que permitan no solo descentralizar el vertimiento de los RC\&D en un solo lugar, sino que le permita al sector informal tener la posibilidad de acudir a ellos. Además de facilitar el manejo y la separación de los materiales potencialmente aprovechables al tener menores volúmenes acopiados en comparación con una escombrera. En la medida en que las prácticas de aprovechamiento en la ciudad se hagan más comunes, estos lugares dejarán de ser estaciones de transferencia para convertirse en estaciones de acopio y tratamiento de los RC\&D.

\section{AGRADECIMIENTOS}

Los autores presentan sus agradecimientos al grupo de investigación materiales compuestos (GMC) de la Universidad del Valle; proyecto VRI C.I. 747 "Reciclar Escombros en Concreto", financiado por la Universidad del Valle, convocatoria 2-2013 de la Vicerrectoría de Investigaciones y al Departamento Administrativo de Ciencia, Tecnología e innovación (COLCIENCIAS) y su programa Jóvenes Investigadores e Innovadores "Virginia Gutiérrez de Pineda", convocatoria 566 de 2012, por su apoyo a esta investigación.

\section{FINANCIAMIENTO}

Esta investigación se realizó en el marco del proyecto VRI C.I. 747 "Reciclar Escombros en Concreto" financiado por la Universidad del Valle, convocatoria 2-2013 de la Vicerrectoría de Investigaciones, y ejecutado por el Grupo de Investigación Materiales Compuestos (GMC) perteneciente al centro de excelencia en nuevos materiales (CENM) de la Universidad del Valle.

\section{REFERENCIAS}

Adam, M., \& Kurama, Y. (2013). Desing of concrete mixtures with recycled concrete aggregates. $\mathrm{ACl}$ Materials Journal, 110(5), 483-493.

Alcaldía de Santiago de Cali (31 de agosto de 2004). Decreto No. 0475 de 2004 "por medio del cual se adopta el plan de gestión integral de residuos sólidos PGIRS del municipio de Santiago de Cali". Recuperado el 23 de mayo de 2013, de Alcaldía de Santiago de Cali: http://www.cali.gov.co

Alcaldía de Santiago de Cali (17 de mayo de 2005). Decreto No. 0291 de 2005 "Por medio del cual se regula la gestión integral de escombros en el municipio de Santiago de Cali". Recuperado el 7 de abril de 2013, de Alcaldía de Santiago de Cali: http:// www.cali.gov.co

Alcaldía de Santiago de Cali (23 de febrero de 2009). Decreto 0059 de 2009 "por medio del cual se adoptan los manuales para la implementación de PGIRS en entidades públicas en el municipio de Santiago de Cali y se dictan otras disposiciones". Recuperado el 21 de junio de 2013, de Alcaldía de Santiago de Cali-Documentos compartidos: http:// www.cali.gov.co

Alcaldía de Santiago de Cali (23 de abril de 2009). Ley 1259 "por medio del cual se reglamenta la aplicación de la ley 1259 de 2008 del comparendo ambiental y se dictan otras disposiciones". Recuperado el 15 de mayo de 2013, de Alcaldía de Santiago de Cali-Proyecto de acuerdo: https://www. concejodecali.gov.co

Alcaldía de Santiago de Cali (23 de abril de 2012). Actualización del estudio de definición de áreas potenciales para la localización de sitios de disposición final de escombros para el municipio de Santiago de Cali. Recuperado el 12 de mayo de 2013, de Alcaldía de Santiago de Cali: http://www.cali. gov.co 
Alcaldía de Santiago de Cali (25 de junio de 2012). Acuerdo No. 0327 de 2012 "por medio del cual se modifica parcialmente el acuerdo 282 de 2009 y se dictan otras disposiciones". Recuperado el 2 de junio de 2013, de Alcaldía de Santiago de CaliBoletín oficial No. 115: www.concejodecali.gov.co

Alcaldía de Santiago de Cali (1 de abril de 2012). Proyecto de acuerdo por medio del cual se adopta e plan de desarrollo 2012-2015 del municipio de Santiago de Cali. Recuperado el 11 de abril de 2013, de Alcaldía de Santiago de Cali: www.planeacion.cali.gov.co

Alcaldía Mayor de Bogotá (6 de agosto de 2002). Decreto 1713 de 2002 "por el cual se reglamenta la ley 142 de 1994, la ley 632 de 2000 y la ley 689 de 2001, en relación con la presentación del servicio público de aseo, y el decreto 2811 de 1974 y la ley 99 de 1993. Recuperado el 3 de junio de 2013, de Alcaldía mayor de Bogotá-Diario oficial 44893: http://www.alcaldiabogota.gov.co

Alcaldía Mayor de Bogotá (25 de abril de 2011). Resolución No. 2397 de 2011 "Por la cual se regula técnicamente el tratamiento y/o aprovechamiento de escombros en el distrito capital". Recuperado el 13 de julio de 2013, de Alcaldía Mayor de Bogotá, D.C: http://www.secretariadeambiente.gov.co

Alcaldía Mayor de Bogotá (4-6 de diciembre de 2012). Gestión y control de los residuos de la construcción y demolición (RCD): Programa basura cero-Escombros cero. Recuperado el 20 de mayo de 2013, de Alcaldía Mayor de Bogotá, D.C: http://ambientebogota.gov.co

Aldana, J., \& Serpell, A. (2012). Topics and tendencies of construction and demolition waste: a meta-analysis. Revista de la construcción, 12(22), 4-16.

Bedoya, C., \& Gonzales, L. (2003). El concreto reciclado con escombros como generador de hábitas urbanos sostenibles en la ciudad como ecosistema semi-cerrado, una utopía cultural (tesis de maestría). Medellín: Universidad Nacional de Colombia, sede Medellín.

Castaño, J.; Rodríguez, R.; Lasso, L.; Goméz, A., y Ocampo, S. (2013). Gestión de residuos de construcción y demolición (RCD) en Bogotá: Perspectivas y limitantes. Revista Tecnura, 17(38), 121-129.

Congreso de Colombia (29 de diciembre de 2000). Ley 632 de 2000 "por la cual se modifican parcialmente las leyes 142, 143 de 1994, 223 de 1995 y 286 de 1996". Recuperado el 2 de agosto de 2013, de Congreso de la Republica -Poder público rama legislativa: http://www.fedevivienda.org.co

Congreso de Colombia (7 de agosto de 2002). Ley 769 de 2002 "por la cual se expide el código nacional de tránsito terrestre y se dictan otras disposiciones". Recuperado el 5 de junio de 2013, de Congreso de Colombia-Diario oficial 44893 y 44932 : http://www.alcaldiabogota.gov.co

Congreso de la República (19 de diciembre de 2008). Ley 1259 de 2008 "por medio de la cual se instaurara en el territorio nacional la aplicación del comparendo ambiental a los infractores de las normas de aseo, limpieza y recolección de escombros; y se dictan otras disposiciones". Recuperado el 10 de julio de 2013, de Diario oficial-Congreso de la República: http://190.147.213.68:8080/homepage/ DIARIO_OFICIAL/2008/47208.pdf

Consejo de Medellín (1 de enero de 2009). Acuerdo Municipal No. 62 de 2009 "por medio del cual se establece una política pública para la gestión de escombros en la ciudad de Medellín". Recuperado el 12 de julio de 2013, de Consejo Municipal de Medellín-Gaceta oficial No. 3596: http://www.medellin.gov.co

DAGMA (10 de noviembre de 2011). Estudio de escombros en la ciudad de Cali. Recuperado el 5 de junio de 2013, de Grupo de control de impactos al suelo; Alcaldía de Santiago de Cali: http://www.cali.gov.co

DAGMA (5 de septiembre de 2012). Marco normativo para el manejo de escombros de demoliciones y construcciónes en la ciudad de Cali. Recuperado el 15 de mayo de 2013, de Alcaldía de Santiago de Cali: www.dagma.gov.co

DAGMA (28 de septiembre de 2013). Dagma sensibilizó el comparendo ambiental en operativos de escombros en las comunas 13 y 21. Recuperado el 2 de octubre de 2013, de Alcaldía de Santiago de 
Cali-Comunicaciones DAGMA: http://www.cali. gov.co/dagma/publicaciones

DAGMA (03 de febrero de 2014). Cali tendrá dos estaciones para la disposición final de escombros, en 2015. Recuperado el 17 de febrero de 2014, de Alcaldía de Santiago de Cali-Comunicaciones DAGMA: http://cali.gov.co/publicaciones

Deshpande, N.; Kulkarni, S., \& Patil, N. (2011). Effectiveness of using coarse recycled concrete aggregate in concrete. International Journal of Earth and Engineering, 4(96), 913-919.

Diario ADN (11 de julio de 2013). Candelaria restringió evacuación de escombros de Cali. Recuperado el 25 de agosto de 2013, de Diario ADN-Cali: http:// diarioadn.co/cali

Diario El Pueblo (07 de septiembre de 2013). Los escombros, un problema crónico en Cali. Recuperado el 20 de septiembre de 2013, de Diario El Pueblo: http://elpueblo.com.co

Eijaiek, M.; Quiñones, E., y Mouthon, B. (2011). Aprovechamiento de los escombros generados en actividades de demolición de placas de pavimento en Cartagena-Colombia. Hacia la sustentabilidad: Los residuos sólidos como fuente de energía y materia prima, 554-562.

El País (01 de marzo de 2008). ¿Hasta cuándo con los escombros? Recuperado el 25 de junio de 2013, de periódico el País: http://historico.elpais.com.co

El Tiempo (13 de abril de 2012). ¿Quién responde por estas montañas de escombros?/ Con nombre propio. Recuperado el 17 de febrero de 2014, de periódico El Tiempo: http://www.eltiempo.com

El Tiempo (05 de septiembre de 2012). Cali busca una salida para sus escombros. Recuperado el 3 de junio de 2013, de periódico El Tiempo: http://www. eltiempo.com

El Tiempo (29 de diciembre de 2013). Bogotá tiene 368 puntos críticos por cuenta de los escombros. Recuperado el 17 de febrero de 2014, de periódico El Tiempo: http://www.eltiempo.com

El Tiempo (04 de julio de 2013). Pretenden acabar con montañas de escombros en Cali. Recuperado el 10 de agosto de 2013, de periódico El Tiempo: http:// www.eltiempo.com
Hincapié, A., y Aguja, E. (2003). Agregado reciclado para mortero. Revista Universidad EAFIT, 39(132), 76-89.

Janssen, G. (2005). General approach to reuse and recycling in construction. Use of Recycled Materials (RILEM TC 198: Final report), 27-32.

Lederle, R., \& Hiller, J. (2013). Reversible shrinkage of concrete made with recycled concrete aggregate and other aggregate types. ACI Materials Journal, 110(4), 423-433.

Limbachiya, M.; Koulouris, A.; Roberts, J., \& Fried, A. (2004). Performance of recycled aggregate concrete. RILEM International Symposium on Environment Conscious Materials, 127-136.

Lin, Y.; Tyan, Y.; Chang, T.-P., \& Chang, C.-Y. (2004). An assessment of optimal mixture for concrete made with recycled concrete aggregates. Cement and Concrete Research (34), 1373-1380.

Malasev, M.; Radonjanin, V., \& Marinkovic, S. (2012). Recycled concrete as aggregate for structural concrete production. Sustainability (2), 1204-1225.

Muller, A. (2005). Overview regarding construction and demolition waste in Germany. Use of Recycled Materials (RILEM TC 198: Final Report), 3-5.

Oikonomou, N. (2005). Recycled concrete aggregates. Cement \& Concrete Composites, 27, 315-318.

Poon, C., \& Chan, D. (2007). The use of recycled aggregate in concrete in Hong Kong. Resources, Conservation and Recycling, 50, 293-305.

Rakshvir, M., y Barai, S. (2006). Estudios sobre hormigón reciclado con áridos. Waste Management \& Research(24), 225-233.

Salem, R.; Burdette, E., \& Jackson, M. (2003). Resistance to freezing and thawing of recycled aggregate concrete. ACI Materials Journal, 100(3), 216-222.

Sánchez, D.; Ruiz, F.; Ortiz, H., \& Silva, M. (25 de agosto de 2013). Infografia: ¿De dónde vienen y dónde van a parar los escombros de Cali? Recuperado el 29 de agosto de 2013, de periódico El País: http:// www.elpais.com.co

Seddik, M.; Ouchagour, Y., \& Limbachiya, M. (2012). Performance of Portland/Silica fuem cement concrete produced with recycled concrete aggregate. ACI Material Journal, 109(1), 91-101. 
Serrano, G., y Ferreira, J. (2009). Aprovechamiento de los escombros para la producción de concreto. Red de ingeniería en saneamiento ambiental (REDISA), 1-10.

Shima, H.; Tateyashiki, H.; Matsuhashi, R., \& Yoshida, Y. (2005). An advanced concrete recycling technology and its applicability assessment trugh input-output analysis. Journal of Advanced Concrete Technology, 3(1), 53-57.

Sriravindrarajah, R.; Wang, N., \& Ervin, L. (2012). Mix desing for previous recycled aggregate concrete. International Journal of Concrete Structures and Materials, 6(4), 239-246.

Thomas, C.; Setién, J.; Polanco, J.; Alaejos, P., \& Sanchez de Juan, M. (2013). Durability of recycled aggregate concrete. Construction and Building Materials(40), 1054-1065.

Torgal, F., \& Jalali, S. (2011). Eco-efficient construction and building materials. Springer Verlag London $\mathrm{Li}$ mited, 51-74.

UAESP (12 de enero de 2012). Escombros cero; Manejo integral de los escombros en la ciudad de Bogotá. Recuperado el 20 de junio de 2013, de Alcaldía Mayor de Bogotá; Unidad administrativa de servicios públicos: http://www.secretariadeambiente. gov.co
Valdéz, G., y Rapimán, J. (2007). Propiedades físicas y mecánicas de bloques de hormigón compuestos con áridos reciclados. Información Tecnológica, 18(3), 81-89.

Vázquez, E. (2005). Overview regarding construction and demolition waste in Spain. Use of Recycled Materials (RILEM TC 198-URM: Final report), 26-26.

Xiao, J.; Li, W., \& Huang, X. (2012). An overview of study on recycled aggregate concrete in China (1996-2011). Construction and Building Materials(31), 364-383.

Xiao, J.; Tawana, M., \& Huang, X. (2012). Review of studies on structural performance of recycled aggregate concrete in China. Science China; Technological Sciences, 55(10), 2727-2739.

Yang, K.; Chung, H., \& Ashour, A. (2008). Influence of type and replacement level of recycled aggregates on concrete properties. ACI Materials Journal, 105(3), 289-297.

Yaprak, H.; Aruntas, H.; Demir, I.; Sissek, O., \& Durmus, G. (2011). Effects of the fine recycled concrete aggregates on the concrete properties. International Journal of the Physical Sciences, 6(10), 2455-2461. 